\title{
VARIABILIDAD INTRAINDIVIDUAL E (IN)DEPENDENCIA EPISTÉMICA DEL DOCENTE EN EL DESARROLLO DE CLASES EXPOSITIVAS Y PARTICIPATIVAS-GUIADAS
}

Intraindividual variability and epistemic (in)dependence of teacher during both expositive and guided-participative classes

\author{
NÉstor D. RoselLi $\left.\right|^{1}$ \\ Pontificia Universidad Católica Argentina, Santa María de los Buenos Aires \\ (Recibido el 17/04/2011 - Aceptado el 02/06/2011)
}

\begin{abstract}
RESUMEN
El objetivo de la presente investigación es comparar el desarrollo epistémico de dos estilos de enseñanza tradicionalmente opuestos: expositivo y participativo-guiado, en un contexto naturalista o de clase real. Específicamente se trata de analizar descriptivamente ciertas diferencias no fácilmente perceptibles entre tipos de clase claramente representativas de dichos estilos. El foco está puesto en el desarrollo instructivo del conocimiento, esto es, en el proceso de enseñanza en sí mismo; no se consideran los efectos derivados en términos de aprendizaje.

Las diferencias "ocultas" que se trata de poner en evidencia conciernen a la estabilidad-variabilidad intraindividual del docente y a la dependencia-independencia de éste respecto de la fuente epistémica.

Participaron cuatro profesores de biología, los cuales debían dar una clase a sus propios alumnos de secundaria sobre un tema extra-curricular, ajustándose dos de ellos a un estilo expositivo y los otros dos a un estilo participativo-guiado.

Los resultados muestran notorias diferencias entre ambos estilos de enseñanza en los aspectos referidos. En efecto, el estilo expositivo exhibe un patrón intraindividual mucho más estable en lo que hace al desarrollo epistémico-didáctico, en tanto que el estilo participativo-guiado muestra mayor variabilidad inter-clases. Por otra parte, el estilo participativo-guiado manifiesta un desarrollo más flexible y libre respecto de la fuente epistémica (texto proporcionado a cada profesor), tanto a nivel de contenido como lingüístico; el estilo expositivo, en cambio, es más fiel a dicha fuente epistémica.
\end{abstract}

Palabras clave: Estilos de enseñanza, clase expositiva, clase participativa, discurso docente, constructivismo.

1 Director Centro de Investigaciones en Psicología y Psicopedagogía de la Pontificia Universidad Católica Argentina, Santa María de los Buenos Aires. E-maill: nestorroselli@uca.edu.ar 


\begin{abstract}
The objective of this research is to compare the epistemic development of two opposite teaching styles: expositive and guided-participative, in a natural setting. The aim was to analyse some non-explicit or hidden differential traits of each style and make them explicit. This analysis is focused on the instructive development of knowledge, that is, the teaching process itself and not the learning outcomes.

These hidden differential traits concern the stability-variability of teaching and the dependence-independence of the teacher from the epistemic.source.

Four biology teachers were invited to teach their own secondary students the same extracurricular subject. It was agreed that two of them would use an expositive style, while the two others implemented a participative style.

Results show great differences between both teaching styles. Expositive style displays a more fixed intraindividual pattern in the epistemic development, while the participative teacher is more variable in the way he teaches the same knowledge. On the other hand, the participative style is more independent from the epistemic source (the scientific text offered to all teachers), while the expositive modality sticks more to the original source.
\end{abstract}

Keywords: Teaching styles, expositive class, participative class, teaching discourse, constructivism.

\title{
INTRODUCCIÓN
}

Los estilos de enseñanza es un tema tradicionalmente presente en la investigación en educación. Es común que una obra general sobre psicología de la instrucción (por ejemplo, Beltrán, 1998) dedique un apartado a este tema, generalmente dentro de la unidad temática "variables del docente", incluyendo además unidades referidas a las "variables del alumno", las "variables del contexto" y la "interacción docente-alumno". El término "estilo de enseñanza” es ciertamente complejo ya que no tiene una semántica unívoca, aunque hay consenso en considerarlo una disposición subjetiva más o menos estable, asentada en rasgos e historias personales, pero también en una particular teoría implícita de la enseñanza y del aprendizaje. Precisamente, esto último es lo que lo diferencia del concepto más técnico "método o estrategia de enseñanza", que implica una concepción explícita de lo que es enseñar y aprender, y de los objetivos buscados. Como dice Carretero, "son numerosas las investigaciones que muestran que el docente tiende a enseñar como efectivamente aprendió y no sólo basándose en las teorías y conocimientos que recibió en su formación" (1998: 19).

Uno de los investigadores más reconocidos del proceso de instrucción escolar, Bennett, distingue (1998) tres grandes líneas en la investigación de los últimos cuarenta años: los estilos de enseñanza, las oportunidades de aprendizaje (referidas al alumno y al contexto) y las tareas en el aula (la interacción enseñante propiamente dicha). Coll y Sánchez (2008), en un reciente número de la emblemática Revista de Educación, editada por el Ministerio de Educación y Ciencias español, también se refieren al creciente interés en el análisis de la interacción alumno-profesor, sobre todo con metodologías cualitativas y con énfasis en el intercambio discursivo. Para estos autores, el enfoque sociocultural y situado de la enseñanza y del aprendizaje, prevaleciente en la actualidad, no se conciliaría mucho con una 
idea descontextualizada del estilo de enseñar. De hecho, justo es reconocerlo, en la última década se ha puesto más énfasis en el estudio de las estrategias de aprendizaje que en el de los estilos o modalidades de enseñanza (Gargallo López, Suárez Rodríguez y Ferreras Remesal, 2007; Marín Gracia, 2002; Ruiz Lara, Hernández Pina y Ureña Villanueva, 2008). Si bien es cierto que hay una estrecha relación entre estrategias de enseñanza y estrategias de aprendizaje (Monereo, 2006), el acento en las segundas va de la mano con la importancia creciente que se otorga a la metacognición y autorregulación cognitivas y al "aprender a aprender". Esto implica disminuir el peso de la determinación docente, o en todo caso, hacer del "enseñar a aprender" la función básica.

Con todo, en esta cuestión corresponde hacer una distinción entre las corrientes europeas y anglosajonas (Carretero, 1998). La ideología prevaleciente en la investigación educativa actual en Europa es el socioconstructivismo vygotskiano, que pone el acento en el proceso comunicacional (discursivo y situado) que se desarrolla en el aula. La corriente anglosajona es más cognitivista, con aproximaciones más empiristas, aún en el caso de investigaciones de corte naturalista. En esta corriente, el estudio de la enseñanza y del comportamiento docente es de interés permanente. De hecho, el término “estilo de enseñanza” es un descriptor insoslayable a la hora de efectuar una revisión bibliográfica actualizada en teoría de la enseñanza y psicología de la instrucción.

Por otra parte, hablar de estilos de enseñanza no implica una visión descontextualizada del hecho educativo. Cuando se habla de estilos en la actualidad no se deja de reconocer la fuerte dependencia de la modalidad enseñante respecto a factores del contexto. La tradición institucional, el número de alumnos, el ámbito físico, el tiempo disponible para el desarrollo didáctico, el tipo de episteme a enseñar, el nivel de experticia del docente, las expectativas de los alumnos, el nivel dentro del sistema educativo, los recursos tecnológicos y didácticos, son, entre otros, condicionantes de la modalidad de enseñanza implementada.

Las tipologías en torno a los estilos de enseñanza con múltiples. Ello es entendible ya que responden a diferentes criterios. Fernández González y Elortegui Escarpín (1996) enumeran los siguientes modelos didácticos: profesor transmisor, profesor tecnológico, profesor artesano y profesor constructor. De manera bastante coincidente, García Pérez (2000) habla de cuatro modelos didácticos: tradicional, tecnológico, espontaneísta y alternativo (investigación en la escuela). Wheeler y Marshall (citado por Pinedo Ávila, 2008) elaboraron un inventario que permite identificar cuatro tipos de instructores: oyente, director, intérprete y entrenador. Por su lado, el investigador francés Astolfi (1997) diferencia tres modelos básicos de enseñanza: tradicional, conductista y constructivista. Refiriéndose específicamente a la enseñanza de la ciencia, Pozo y Gómez (1998) enumeran estos tipos de enseñanza, que más que estilos aluden a estrategias o modalidades didácticas: tradicional, por descubrimiento, expositiva, por conflicto cognitivo, por investigación dirigida, y por explicación y contrastación de modelos. Más centrado en los aspectos psicosociales del liderazgo docente, Berkovitz (citado por Leyens, 1989) ha distinguido entre una modalidad focalizada en la realización de la tarea ("técnica") y otra con acento en las relaciones humanas y la cohesión grupal ("mantenimiento"), distinción retomada por Yildirim, Acar, Bull y Sevinc (2008), quienes diferencian entre el "estilo orientado a la gente" y el "estilo orientado a la tarea". 
A pesar de la diversidad, si se trata de simplificar el espectro de estilos y modalidades propuestas, la pluralidad podría acotarse al reconocimiento de dos tipos básicos. Una distinción muy común en los ámbitos educativos es entre un llamado enfoque conductista de la enseñanza y su opuesto: el omnipresente constructivismo. En verdad, cuando se menciona el término conductismo en este contexto no se lo usa en el sentido estricto de la corriente psicológica anglosajona, sino más bien en el de una concepción de la enseñanza de "afuera - adentro", donde el peso de la actividad recae en el docente, que debe "transmitir" al alumno el conocimiento. El constructivismo, en cambio, respondería a una concepción de la enseñanza de "adentro - afuera", que prioriza la actividad del alumno y la "construcción" que hace del conocimiento (con la ayuda del docente).

Esta distinción, simplista por cierto, se reitera en los medios académicos y científicos. Así, se suele diferenciar entre modalidades de enseñanza "tradicionalistas" y "progresivistas" (González Peiteado, 2009; Kozulin, 2004). Bennett (1979), por ejemplo, identifica doce estilos de enseñanza dentro del continuum progresista-tradicional. En un polo se ubican los profesores que enfatizan la integración interdisciplinaria, la motivación intrínseca, la actividad de los alumnos, el agrupamiento flexible, la participación de los estudiantes en la planificación del curriculum, y cierta despreocupación del docente por el control de la clase y del rendimiento. En el polo opuesto está el profesor centrado en su disciplina, que acentúa la motivación extrínseca y el control del rendimiento, que promueve la lección magistral y el agrupamiento fijo de los alumnos (clase total y trabajo individual). Entre ambos extremos se sitúan una serie de tipos mixtos.

En la misma línea, Flanders (1960) distingue entre un estilo directo de enseñanza, caracterizado por la exposición de las propias ideas del docente, y un estilo indirecto, donde se promueven las ideas de los alumnos a través del diálogo.

Atendiendo al tipo de liderazgo docente, Anderson y Gordon (Weber, 1976) reconocen dos estilos básicos: dominador e integrador, que se corresponden con las formas clásicas autocrática y democrática. En la misma línea, Coldren y Hively (2009) hablan de "authoritarian and authoritative styles". La misma dicotomía es enunciada en términos de estilo activo versus estilo pasivo por Michel, James y Varela (2009), método de enseñanza activo versus tradicional (Weltman y Whiteside, 2010), liderazgo "transformational" versus "transactional" por Hood, Poulson, Mason, Walker y Dixon (2009), orientación centrada en la enseñanza versus orientación centrada en el aprendizaje por Gargallo López (2008), interacción docente-alumno evaluativa versus co-constructiva (Toczek y Morge, 2009), "controlling style" versus "autonomy-supportive style" (Reeve, 2009), "directed tutorial style" versus "inductive tutorial style" (Jones, Holland y Oldmeadow, 2008), aprendizaje intrapsicológico versus aprendizaje intersubjetivo o dialógico (Aubert, García y Racionero, 2009) y, finalmente, instrucción versus construcción según una formulación recurrente en los ámbitos académicos.

El estilo de enseñanza es un constructo multidimensional. De hecho, cada una de estas formulaciones pone el acento en un aspecto determinado: la distribución del poder y de la influencia (concentrados en el docente o distribuidos entre todos los participantes del sistema), la actividad productora del conocimiento (clase expositiva o clase participativa con andamiaje docente), el método de trabajo (individual o por grupos), la intensidad 
y direccionalidad del intercambio docente-alumnos y alumno-alumno (baja o alta interactividad), las características cualitativas de la interacción (incluyendo la actitud física: gestos, mirada, tono de voz y desplazamiento físico) y el involucramiento afectivo y el clima de trabajo (ambiente neutro o afectivamente cálido). Incluso algunas tipologías con explícitamente multidimensiones, como la distinción entre estilo tradicional y progresista que hacen Bennett (1979) y González Peiteado (2009). Esto genera una dificultad desde el punto de vista metodológico, sobre todo en las aproximaciones naturalísticas, ya que no hay tipos puros. La tentación es la de renunciar al constructo como tal y realizar abordajes dimensionales episódicos. Es lo que hacen, por ejemplo, Coll, Onrubia y Maurí (2008) cuando analizan secuencias discursivas episódicas de tipo expositivo, al interior de una clase; es cierto que hay momentos didácticos expositivos en clases muy diferentes, pero también es cierto que la clase magistral es una unidad tipológicamente reconocible como tal, y no sólo por el método expositivo de la información empleado por el docente. Constituye claramente un estilo de enseñanza.

Algunas posturas van más lejos, vinculando estos estilos en pugna con diferentes paradigmas epistemológicos. Para Días Arce (2003), por ejemplo, el tema de la transposición didáctica, o sea la transformación del objeto de saber en objeto de enseñanza, no es un mero problema de modalidades de enseñanza; enfrenta una pedagogía lineal a una pedagogía dialógica, que en última instancia remite a distintas concepciones de lo que es el conocimiento: una reproducción de la realidad o una construcción social de ella. Para el socio-constructivismo, entendiéndolo como paradigma amplio que basa el conocimiento en la intersubjetividad, está claro que la enseñanza es un acto comunicativo de creación de significados compartidos; para posiciones no-constructivistas la enseñanza es básicamente instrucción, esto es, transmisión de significados (conocimiento epistémico que se supone exterior al hecho educativo y del cual, se supone, el docente es el fiel traductor).

La forma empírica más representativa del paradigma socio-constructivista es la clase dialogada con activo apuntalamiento docente en la zona de desarrollo próximo (participación guiada o andamiada, en la que el docente tiene una función mediadora o facilitadora del aprendizaje). Como lo que interesa es la construcción intersubjetiva del significado, las investigaciones analizan sobre todo el intercambio discursivo entre el docente y los alumnos, pudiendo llegar a establecer ciertos patrones diferenciales asociados a determinadas variables. Menos atención reciben los efectos cognitivos (individuales) de aprendizaje, especialmente en relación a los obtenidos con prácticas de enseñanza alternativas. Corresponde aclarar que el tan en boga "aprendizaje colaborativo", que enfatiza la interacción sociocognitiva entre pares, tiene vínculos teóricos muy estrechos con el socio-constructivismo (Roselli, 2007); de todas maneras no es su núcleo duro, como sí lo es el andamiaje docente de la construcción intersubjetiva del grupo-clase. Al respecto, Onrubia (1996) define a la enseñanza como "proceso de creación de Zonas de Desarrollo Próximo y de asistencia en ellas" ("ayuda ajustada") (p. 102). De lo que se trata es de montar la influencia experta (garante del conocimiento nuevo emergente) en el saber pre-existente de los alumnos.

En cuanto al paradigma instructivo (no-socioconstructivista), la clase expositiva es la expresión empírica que mejor lo representa y que recibió una cierta atención en la 
investigación didáctica -hegemónicamente socio-constructivista-, aunque a veces concebido como una forma de discurso más que como un estilo de enseñanza.

La clase expositiva tuvo en Ausubel su gran defensor, sobre todo frente a la idea de autoestructuración y aprendizaje por descubrimiento. Como cabeza de la corriente conocida como aprendizaje significativo, este autor (1983) reivindica el modelo didáctico de transmisión-recepción o expositivo, aunque lo diferencia absolutamente de la forma tradicional, mecánica, memorística y repetitiva. Para que haya un genuino aprendizaje (memoria comprensiva) deben darse dos condiciones de significatividad: coherencia lógica estructural y secuencial del material (significatividad lógica) y adaptación de éste a la estructura cognitiva y al saber previo del sujeto que aprende (significatividad psicológica).

Se debe insistir, tal como lo hace Cubero (2005), en que la dimensión recepción-descubrimiento es independiente de la dimensión repetitivo-significativo, y no puede ser confundida con ella. Una clase magistral implica una actitud receptiva por parte del alumno, pero esto no desemboca necesariamente en un saber repetitivo o no-significativo, del mismo modo que el aprendizaje por descubrimiento no asegura significatividad. Esto es útil a la hora de evaluar la calidad (buena o mala) de una clase magistral y de una clase constructiva.

También se debe tener en cuenta que la modalidad de enseñanza expositiva no es sinónimo de clase magistral, es decir, del desarrollo oral de un tema por parte del docente. Quaas y Crespo (2003) distinguen además la modalidad expositiva-demostrativa, característica de las ciencias básicas, donde el docente lleva adelante demostraciones experimentales acompañadas de comentarios explicativos. Lo importante en esto es que el desarrollo epistémico está a cargo del docente.

Hay que precisar que en los últimos tiempos han aparecido críticas fuertes a las modalidades de enseñanza de mínima intervención docente: aprendizaje por descubrimiento, aprendizaje basado en problemas, y aprendizaje experiencial, definidas en general como constructivistas (Kirschner, Sweller y Clark, 2006). Es cierto que esto no le cabe tanto al socio-constructivismo que reivindica la necesidad de la guía y el andamiaje permanente por parte del docente, sino sobre todo a las posturas que reducen la intervención epistémica del docente al mínimo. Tales críticas conciernen a los efectos en términos de aprendizaje. De manera general, las modalidades de enseñanza centradas en el alumno no conducirían a un mayor aprendizaje que el logrado con métodos tradicionales; incluso los resultados serían peores. Cada vez más frecuentemente aparecen reportes de investigaciones que reivindican el valor de la clase magistral (Schwerdt y Wuppermann, 2010; Stacy, 2009) o que ponen en duda la pretendida eficacia de las modalidades "activas" o "constructivas" de enseñanza (Cardozo, Andino, Brunnetti Esquivel y Espindola, 2008; Quaas y Crespo, 2003; Weltman y Whiteside, 2010; cfr. además las múltiples enumeraciones que realizan Kirschner et al., op. cit.).

Sin embargo, es justo reconocer que, coexistiendo con estas publicaciones críticas, hay también otras que presentan resultados favorables a las formas de enseñanza no-tradicionales (como las ya citadas de Toczek y Morge, 2009, y la de Reeve, 2009). En verdad, es bastante contradictorio el panorama que existe en este aspecto, lo cual reclama investigaciones más afinadas y discriminantes, no tanto de la eficacia en bloque, sino de los efectos múltiples y diversos, que apunten a características específicas y no a consecuencias generales. 
De todas maneras, no es objeto de la presente investigación considerar los efectos o resultados de aprendizaje. El objetivo es analizar descriptivamente diferencias cualitativas que se registran en el desarrollo mismo de la enseñanza. Quizás este es el defecto más recurrente de la investigación experimental y cuasi-experimental en el campo de la enseñanza: se pone todo el peso en el análisis de los efectos pero se descuida al análisis del proceso de enseñanza en sí mismo, sobre todo de aspectos no tan visibles ni explícitos. Precisamente, el análisis del discurso docente y de la comunicación en el aula trata de llenar este vacío. En las dos últimas décadas el análisis de los procesos comunicacionales en escenarios educativos naturales ha adquirido una fuerte presencia, especialmente en el continente europeo.

Es en este contexto que se ubica el presente estudio, dirigido a poner en evidencia dos aspectos ocultos del proceso de enseñanza: la estabilidad-variabilidad del docente en el desarrollo epistémico de un mismo tema a diferentes grupos de alumnos, y la dependenciaindependencia del desarrollo epistémico que hace el docente en sus clases respecto de la fuente epistémica externa (texto-fuente). Lo distintivo del encuadre metodológico propuesto es que, si bien se preservan las condiciones naturales de enseñanza, estas se adecuan a un dispositivo cuasi-experimental mínimo para posibilitar la comparación de los casos considerados. Además, el análisis del discurso que se propone no se queda en una hermenéutica libre, sino que recurre a niveles de formalización precisos.

\section{MÉTODO}

\section{Participantes}

Se seleccionaron cuatro profesores de Biología de cuatro colegios secundarios, dos públicos y dos privados. A pesar de las diferencias, ninguno de los establecimientos tenía características especiales o excepcionales. Los criterios de selección fueron los siguientes: todos debían tener a su cargo un grupo de alumnos de primer año (13 años), todos debían ser considerados "buenos profesores" por estos, todos debían aceptar colaborar con la investigación y, finalmente, dos de ellos debían usar habitualmente una modalidad de trabajo altamente participativa, en tanto que los otros dos debían naturalmente ser más tradicionales o expositivos en la manera de enseñar. Por supuesto, este último criterio no apuntó a buscar los casos paradigmáticos de los estilos que se querían comparar (cosa imposible, por cierto), sino a seleccionar docentes que pudieran sentirse cómodos (no forzados) con la modalidad que explícitamente cada uno debía implementar. Debe también aclararse que los docentes colaboraron "desde dentro" de la investigación, aunque el detalle de los aspectos que formaban parte del análisis comparativo sólo se explicitó, de común acuerdo, una vez realizadas las clases.

Los alumnos de cada grupo (14), fueron seleccionados aleatoriamente de entre el grupo-clase habitual de cada profesor, que en general duplicaba esa cifra. En rigor, si bien estas clases fueron las utilizadas como base del análisis, se le pidió a cada profesor que reiterara la clase para el resto de los alumnos de su grupo real. 


\section{Material}

El tema objeto de enseñanza ("Las aves") era extracurricular. A partir de la consulta a manuales y a especialistas, se elaboró un texto-fuente epistémico común. Estaba estructurado en función de los siguientes temas y subtemas:

1. Las plumas: tipos y funciones

2. Por qué vuelan las aves: explicación física del mecanismo de vuelo

2a. El aire como materia

2b. Interacción entre el gas aire y el sólido ave

2c. Rasgos anatómicos que favorecen el vuelo

2d. Física del vuelo batido

2e. Física del vuelo planeado

3. Clasificación de las aves según su alimentación

4. La adaptación morfológica al medio

5. La adaptación dinámica al medio

El punto 2 es el de mayor extensión y complejidad conceptual (incluye varios subtemas). Los puntos 1 y 3 conciernen a aspectos fácticos o descriptivos. Los restantes son de naturaleza conceptual.

El cuestionario de evaluación del aprendizaje o post-test (el mismo para todas las clases) estaba compuesto por preguntas abiertas referentes al conocimiento específico enseñado, además de otras de extensión y transferencia. De todas maneras, estos datos escapan al objetivo del presente reporte, dedicado al análisis descriptivo del proceso de enseñanza y no a los logros de aprendizaje. Por idéntico motivo, tampoco se aludirá a los resultados del cuestionario de evaluación del nivel de conocimiento previo o pre-test.

\section{Procedimiento}

La consigna era bastante simple. Después de un pre-test individual aplicado a los alumnos de los grupos constituidos, cada profesor debía dar una clase de 50 minutos a su respectivo grupo sobre el tema mencionado, la cual debía ajustarse al texto-fuente epistémico que le era entregado con una semana de antelación. El resto de los alumnos debía realizar un trabajo práctico de la materia en otro salón o la biblioteca, con la supervisión de colaboradores en la investigación. La división del grupo natural en dos subgrupos obedeció a la necesidad de facilitar el registro verbal y de imagen del proceso enseñante y el interrogatorio individual de cada alumno, realizado por los integrantes del equipo de investigación, sobre las respuestas escritas en el cuestionario final de evaluación. A dos de los profesores (Nancy y Ana) se les pidió que dieran una clase con un estilo claramente expositivo, debiendo el docente poner el acento en una buena explicación de los temas; a los otros dos (Inés y Carlos) se les solicitó una clase altamente participativa y dialogada, debiendo poner énfasis en guiar 
adecuadamente el aprendizaje a partir de los aportes de los alumnos. Con esto se buscaba diferenciar claramente los dos estilos, evitando modalidades híbridas. Salvo esta consigna, formulada en términos bien generales, los profesores disponían de libertad para realizar el desarrollo docente de la clase. Había dos restricciones adicionales. Independientemente de cualquier evaluación que quisiera hacer el docente, habría una evaluación final externa que consistiría en una serie de preguntas abiertas sobre los temas del texto-fuente, a desarrollar individualmente. La implementación de una evaluación estandarizada era un requisito metodológico esencial para asegurar la comparabilidad de los efectos de aprendizaje logrados por las distintas clases. Otra restricción era que los docentes debían abstenerse de implementar técnicas de trabajo grupal, ello por una razón práctica (se debía garantizar el registro de la comunicación oral) y de diseño (estaba prevista una futura comparación con una modalidad de enseñanza basada en el aprendizaje colaborativo o entre pares).

Al término de cada clase, el profesor administraba un cuestionario abierto de evaluación del conocimiento aprendido. Mientras los alumnos lo completaban, supervisados por colaboradores, el docente reiteraba la clase para el resto de los alumnos de su grupo real. Todas las clases fueron video y audiograbadas.

\section{RESULTADOS}

\section{Estabilidad-variabilidad intraindividual}

En primer lugar, interesaba constatar si había diferencia en cuanto a la estabilidad del patrón enseñante según el estilo docente. Empíricamente, esto supone comparar la constancia-variabilidad del desarrollo epistémico en las dos clases que tuvo a su cargo cada docente. Esta comparación se realizó en relación a la secuencia del abordaje subtemático de dos temas específicos: descripción de los tipos y funciones de las plumas, y explicación física del vuelo de las aves ("por qué las aves vuelan").

\section{DESCRIPCIÓN DE LOS TIPOS Y FUNCIONES DE LAS PLUMAS}

El texto-fuente proporcionado a cada docente respondía a la siguiente estructura epistémica (Esquema 1). El desarrollo secuencial del mismo era en sentido horizontal: Aa, Ab, Ac, $\mathrm{Ad}, \mathrm{Ba}, \mathrm{Bb}, \mathrm{Bc}, \mathrm{Bd}, \mathrm{Ca}, \mathrm{Cb}, \mathrm{Cc}, \mathrm{Cd}$.

\begin{tabular}{lllll}
\hline & Nombre a & Localización b & Morfología c & Función d \\
\hline A & Pennas & Ala/Cola & Largas/Duras & Remos/Timón \\
B & Tectrices & Dorso & Medianas/Semiduras & Protección \\
C & Plumones & Pecho & Pequeñas/Blandas & Temperatura \\
\hline
\end{tabular}

ESQUEMA 1. Estructura del tema “Tipos y funciones de las plumas” según el texto-fuente. 
La Tabla 1 presenta el desarrollo secuencial realizado por cada docente. Se tiene en cuenta el orden de introducción del concepto y no las reiteraciones del mismo. Si se otorga 1 punto a las distancias mínimas macro (mayúsculas) y microtemáticas (minúsculas) entre las clases 1 y 2 de cada profesor, se obtiene una medida general de la distancia o variabilidad inter-clases, que corresponde a la sumatoria de las distancias parciales.

TABLA 1. Desarrollo secuencial subtemático realizado por los 4 profesores en las 2 clases dictadas y puntaje de distancia entre las mismas.

\begin{tabular}{|c|c|c|c|c|c|c|c|c|c|c|c|c|}
\hline & \multicolumn{3}{|c|}{ Nancy (exp.) } & \multicolumn{3}{|c|}{ Ana (exp.) } & \multicolumn{3}{|c|}{ Carlos (part.) } & \multicolumn{3}{|c|}{ Inés (part.) } \\
\hline & $\mathrm{Cl} .1$ & $\mathrm{Cl} .2$ & Dist. & Cl.1 & $\mathrm{Cl} .2$ & Dist. & Cl.1 & $\mathrm{Cl} .2$ & Dist. & Cl.1 & $\mathrm{Cl} .2$ & Dist. \\
\hline & $\mathrm{Aa}$ & Ac & 2 & Ac & $\mathrm{Ac}$ & 0 & Ac & $\mathrm{Ab}$ & 1 & Ac & $\mathrm{Ab}$ & 1 \\
\hline & $\mathrm{Ac}$ & $\mathrm{Ab}$ & 1 & $\mathrm{Ab}$ & $\mathrm{Ab}$ & 0 & $\mathrm{Ab}$ & $\mathrm{Ac}$ & 1 & $\mathrm{Ab}$ & Ac & 1 \\
\hline & $\mathrm{Ab}$ & $\mathrm{Aa}$ & 1 & $\mathrm{Aa}$ & $\mathrm{Bc}$ & 3 & $\mathrm{Aa}$ & $\mathrm{Aa}$ & 0 & $\mathrm{Bb}$ & Aa & 2 \\
\hline & $\mathrm{Ad}$ & $\mathrm{Ad}$ & 0 & Ad & $\mathrm{Bb}$ & 3 & $\mathrm{Cb}$ & $\mathrm{Ad}$ & 4 & $\mathrm{Cc}$ & $\mathrm{Bb}$ & 2 \\
\hline & $\mathrm{Ba}$ & $\mathrm{Bb}$ & 1 & $\mathrm{Bc}$ & $\mathrm{Cc}$ & 1 & $\mathrm{Cc}$ & $\mathrm{Bb}$ & 2 & $\mathrm{Cb}$ & $\mathrm{Ba}$ & 2 \\
\hline & $\mathrm{Bb}$ & $\mathrm{Bc}$ & 1 & $\mathrm{Bb}$ & $\mathrm{Cb}$ & 1 & $\mathrm{Ca}$ & $\mathrm{Bc}$ & 3 & $\mathrm{Ad}$ & $\mathrm{Cb}$ & 4 \\
\hline & $\mathrm{Bc}$ & $\mathrm{Bd}$ & 1 & $\mathrm{Ba}$ & $\mathrm{Aa}$ & 1 & $\mathrm{Bb}$ & $\mathrm{Ba}$ & 1 & $\mathrm{Bc}$ & $\mathrm{Cc}$ & 1 \\
\hline & $\mathrm{Bd}$ & $\mathrm{Ba}$ & 3 & $\mathrm{Bd}$ & $\mathrm{Ad}$ & 1 & $\mathrm{Bc}$ & $\mathrm{Bd}$ & 1 & $\mathrm{Bd}$ & $\mathrm{Ca}$ & 4 \\
\hline & $\mathrm{Ca}$ & $\mathrm{Cb}$ & 1 & $\mathrm{Cc}$ & $\mathrm{Ba}$ & 3 & $\mathrm{Bd}$ & $\mathrm{Cb}$ & 3 & $\mathrm{Cd}$ & Ad & 2 \\
\hline & $\mathrm{Cb}$ & $\mathrm{Cc}$ & 1 & $\mathrm{Cb}$ & $\mathrm{Bd}$ & 3 & $\mathrm{Ad}$ & $\mathrm{Cc}$ & 3 & $\mathrm{Ca}$ & $\mathrm{Bc}$ & 3 \\
\hline & $\mathrm{Cc}$ & $\mathrm{Cd}$ & 1 & $\mathrm{Cd}$ & $\mathrm{Ca}$ & 3 & $\mathrm{Ba}$ & $\mathrm{Cd}$ & 4 & $\mathrm{Aa}$ & $\mathrm{Bd}$ & 4 \\
\hline & $\mathrm{Cd}$ & $\mathrm{Ca}$ & 3 & $\mathrm{Ca}$ & $\mathrm{Cd}$ & 3 & $\mathrm{Cd}$ & $\mathrm{Ca}$ & 3 & $\mathrm{Ba}$ & $\mathrm{Cd}$ & 4 \\
\hline Total & & & 16 & & & 22 & & & 26 & & & 30 \\
\hline & \multicolumn{6}{|c|}{ Total expositivo: 38} & \multicolumn{6}{|c|}{ Total participativo: 56} \\
\hline
\end{tabular}

Nota: La escala de distancia inter-clases para cada docente va de 0 a 72 puntos.

Resulta evidente la mayor variabilidad secuencial del desarrollo epistémico del estilo participativo-guiado. En cambio, el estilo expositivo supone un patrón más constante en el desarrollo didáctico. Las diferencias saltan a la vista aún limitando el análisis a las distancias macrotemáticas (mayúsculas); incluso se hacen más acentuadas cuando se otorga un mayor peso a estas distancias (duplicando el puntaje, por ejemplo).

\section{Explicación física del vuelo de las aves}

El texto fuente planteaba una secuencia explicativa en 7 pasos:

A. El orden físico es universal: todas las cosas materiales, sean seres vivos o no, están sometidas a las mismas leyes físicas. Así, hay una identidad entre el principio explicativo de por qué vuela un ave, un avión, un barrilete, un globo, un dirigible.

B. La materia puede tener 3 estados: sólido, líquido o gaseoso. El aire es un gas. Explicar el vuelo es analizar la interrelación entre un cuerpo sólido y un cuerpo gaseoso que lo rodea. 
C. Apoyarse en el aire es más difícil que apoyarse en una superficie sólida, debido a la particular estructura material del aire. Los fluidos (los líquidos y los gases lo son) tienen una estructura molecular más flexible, libre e informe que los sólidos.

D. El ave tiene características anatómicas que lo hacen apto para el desenvolvimiento en un medio aéreo.

E. Hay que distinguir entre el batido o aleteo y el planeo. Mecánica del aleteo: toda fuerza determina una contrafuerza equivalente (principio de acción-reacción).

F. En el aleteo este juego de fuerzas se traduce en una diferencia de presión (peso del aire) como consecuencia de la compresión del aire inferior (aumento de la presión de éste sobre el animal) y la descompresión del aire superior.

G. Mecánica del planeo: la forma aerodinámica del fuselaje y de las alas hace que el aire circule a mayor velocidad en la parte superior del móvil, generando menor presión del aire respecto del de la parte inferior.

La Tabla 2 presenta el desarrollo docente secuencial de las dos clases de cada profesor y el puntaje de distancia entre ambas. También aquí se tiene en cuenta la introducción y tratamiento específico de cada subtema y no las reiteraciones o alusiones retrospectivas a los mismos. En los casos de omisión de algún subtema en una de las clases la distancia se calcula tomando como referencia el subtema consecutivo.

TABLA 2. Desarrollo secuencial subtemático realizado por los 4 profesores en las 2 clases dictadas y puntaje de distancia entre las mismas.

\begin{tabular}{cccccccccccccc}
\hline & \multicolumn{3}{c}{ Nancy (exp.) } & \multicolumn{3}{c}{ Ana (exp.) } & \multicolumn{3}{c}{ Carlos (part.) } & \multicolumn{3}{c}{ Inés (part.) } \\
\hline Cl. 1 & Cl.2 & Dist. & Cl.1 & Cl.2 & Dist. & Cl.1 & Cl.2 & Dist. & Cl.1 & Cl.2 & Dist. \\
\hline A & --- & 1 & A & A & 0 & A & A & 0 & --- & --- & 0 \\
B & B & 0 & C & B & 1 & B & C & 1 & B & C & 1 \\
C & C & 0 & B & C & 1 & C & B & 1 & C & B & 1 \\
& D & D & 0 & D & D & 0 & E & D & 1 & D & D & 0 \\
& E & E & 0 & E & F & 1 & --- & F & 1 & F & F & 0 \\
& F & F & 0 & F & E & 1 & G & E & 2 & G & E & 2 \\
& G & G & 0 & G & G & 0 & D & G & 3 & E & G & 2 \\
\hline Total & 1 & \multicolumn{1}{c}{ Total expositivo: 5 } & 4 & \multicolumn{1}{c}{ Total participativo: 15 } & \\
\hline \multicolumn{11}{c}{}
\end{tabular}

Nota: La escala de distancia inter-clases para cada docente va de 0 a 24 puntos.

Los datos muestran con claridad que el estilo participativo-guiado es mucho más variable en el orden secuencial del desarrollo temático, no obstante tratarse de un conocimiento explicativo que, a diferencia del descriptivo, tiene una secuenciación lógica más cerrada. Es cierto que se constatan diferencias entre los docentes: la variabilidad es sobre todo 
marcada en Carlos, bastante más que en su colega participativa Inés. De todos modos, los dos docentes expositivos coinciden en tener un patrón de desarrollo didáctico más estable.

\section{Dependencia-independencia respecto de la fuente epistémica (texto fuente)}

\section{Orden secuencial de subtemas}

En las Tablas 3 y 4 se muestran los valores de las distancias sucuenciales entre el desarrollo temático de las clases de cada profesor y el del texto-fuente, correspondientes a los temas "Descripción de los tipos y funciones de las plumas" y "Explicación física del vuelo de las aves". Hay que tener en cuenta que, tratándose de un conocimiento explicativo, la secuencialidad del segundo responde a una lógica más cerrada (la descripción es intrínsecamente más libre); por ello, las diferencias en la explicación del fundamento físico del vuelo tienen un peso mayor.

TABLA 3. Distancia entre el desarrollo secuencial subtemático del texto-fuente y el realizado por los 4 profesores en las 2 clases dictadas sobre el tema "Tipos y funciones de las plumas".

\begin{tabular}{|c|c|c|c|c|c|c|c|c|c|}
\hline & \multirow[t]{2}{*}{$\begin{array}{l}\text { Texto- } \\
\text { fuente }\end{array}$} & \multicolumn{2}{|c|}{ Nancy (E) } & \multicolumn{2}{|c|}{ Ana (E) } & \multicolumn{2}{|c|}{ Carlos (P) } & \multicolumn{2}{|c|}{ Inés $(\mathrm{P})$} \\
\hline & & Cl.1 D & Cl.2 D & Cl.1 D & Cl.2 D & Cl.1 D & Cl.2 D & Cl.1 D & Cl.2 D \\
\hline & $\mathrm{Aa}$ & Aa 0 & Ac 2 & Ac 2 & Ac 2 & Ac 2 & $\mathrm{Ab} 1$ & Ac 2 & $\mathrm{Ab} 1$ \\
\hline & $\mathrm{Ab}$ & Ac 1 & $\mathrm{Ab} 0$ & $\mathrm{Ab} 0$ & $\mathrm{Ab} 0$ & $\mathrm{Ab} 0$ & Ac 1 & $\mathrm{Ab} 0$ & Ac 1 \\
\hline & Ac & $\mathrm{Ab} 1$ & Aa 2 & Aa 2 & $\mathrm{Bc} 1$ & Aa 2 & Aa 2 & $\mathrm{Bb} 2$ & Aa 2 \\
\hline & $\mathrm{Ad}$ & Ad 0 & Ad 0 & Ad 0 & $\mathrm{Bb} 3$ & $\mathrm{Cb} 4$ & Ad 0 & Cc 3 & $\mathrm{Bb} 3$ \\
\hline & $\mathrm{Ba}$ & $\mathrm{Ba} 0$ & $\mathrm{Bb} 1$ & Bc 2 & Cc 3 & Cc 3 & $\mathrm{Bb} 1$ & $\mathrm{Cb} 2$ & $\mathrm{Ba} 0$ \\
\hline & $\mathrm{Bb}$ & $\mathrm{Bb} 0$ & Bc 1 & $\mathrm{Bb} 0$ & $\mathrm{Cb} 1$ & $\mathrm{Ca} 2$ & $\mathrm{Bc} 1$ & Ad 3 & $\mathrm{Cb} 1$ \\
\hline & $\mathrm{Bc}$ & $\mathrm{Bc} 0$ & $\mathrm{Bd} 1$ & $\mathrm{Ba} 2$ & Aa 3 & $\mathrm{Bb} 1$ & $\mathrm{Ba} 2$ & $\mathrm{Bc} 0$ & Cc 1 \\
\hline & $\mathrm{Bd}$ & $\mathrm{Bd} 0$ & $\mathrm{Ba} 3$ & $\mathrm{Bd} 0$ & Ad 1 & $\mathrm{Bc} 1$ & $\mathrm{Bd} 0$ & $\mathrm{Bd} 0$ & $\mathrm{Ca} 4$ \\
\hline & $\mathrm{Ca}$ & $\mathrm{Ca} 0$ & $\mathrm{Cb} 1$ & Cc 2 & $\mathrm{Ba} 1$ & $\mathrm{Bd} 4$ & $\mathrm{Cb} 1$ & $\mathrm{Cd} 3$ & Ad 5 \\
\hline & $\mathrm{Cb}$ & $\mathrm{Cb} 0$ & Cc 1 & $\mathrm{Cb} 0$ & $\mathrm{Bd} 3$ & Ad 4 & Cc 1 & $\mathrm{Ca} 1$ & Bc 2 \\
\hline & $\mathrm{Cc}$ & Cc 0 & $\mathrm{Cd} 1$ & $\mathrm{Cd} 1$ & $\mathrm{Ca} 2$ & Ba 3 & $\mathrm{Cd} 1$ & Aa 4 & Bd 2 \\
\hline & $\mathrm{Cd}$ & $\mathrm{Cd} 0$ & Ca 3 & Ca 3 & $\mathrm{Cd} 0$ & $\mathrm{Cd} 0$ & Ca 3 & $\mathrm{Ba} 4$ & $\mathrm{Cd} 0$ \\
\hline \multirow[t]{2}{*}{ Total } & & 2 & 16 & 14 & 20 & 26 & 14 & 24 & 22 \\
\hline & & \multicolumn{4}{|c|}{ Total expositivo: 52} & \multicolumn{4}{|c|}{ Total participativo: 86} \\
\hline
\end{tabular}

Nota: La escala de distancia clase-texto fuente para cada clase va de 0 a 72 puntos. 
TABLA 4. Distancia entre el desarrollo secuencial subtemático del texto-fuente y el realizado por los 4 profesores en las 2 clases dictadas sobre el tema "Explicación física del vuelo de las aves".

\begin{tabular}{cccccccccc}
\hline $\begin{array}{c}\text { Texto- } \\
\text { fuente }\end{array}$ & \multicolumn{2}{c}{ Nancy (E) } & \multicolumn{2}{c}{ Ana (E) } & \multicolumn{2}{c}{ Carlos (P) } & \multicolumn{2}{c}{ Inés (P) } \\
\hline & Cl.1 D & Cl.2 D & Cl.1 D & Cl.2 D & Cl.1 D & Cl.2 D & Cl.1 D & Cl.2 D \\
\hline A & A 0 & ---1 & A 0 & A 0 & A 0 & A 0 & ---1 & -- 2 \\
B & B 0 & B 0 & C 1 & B 0 & B 0 & C 1 & B 0 & C 1 \\
& C & C 0 & C 0 & B 1 & C 0 & C 0 & B 1 & C 0 & B 1 \\
& D & D 0 & D 0 & D 0 & D 0 & E 1 & D 0 & D 0 & D 0 \\
& E & E 0 & E 0 & E 0 & F 1 & ---2 & F 1 & F 1 & F 1 \\
& F & F 0 & F 0 & F 0 & E 1 & G 1 & E 1 & G 1 & E 1 \\
& G & G 0 & G 0 & G 0 & G 0 & D 3 & G 0 & E 2 & G 0 \\
\hline Total & & 0 & 1 & 2 & 2 & 7 & 4 & 5 & 6 \\
\hline
\end{tabular}

Nota: La escala de distancia clase-texto fuente para cada clase va de 0 a 24 puntos.

Los valores muestran que, en los dos temas analizados, las distancias secuenciales son mucho menores en el caso de los profesores expositivos, lo que significa que el desarrollo de dichos temas en las clases se ajustó estrechamente al desarrollo propuesto por el textofuente. Los docentes participativos, en cambio, se mostraron más independientes en este sentido, muy probablemente porque el acento estaba puesto en la interacción con los alumnos y en la particular situación de intercambio que se generaba. Esto implicaría una adecuación de los contenidos epistémicos a las vicisitudes de las situaciones de interacción docente-alumnos. Esta preocupación por basar el desarrollo didáctico en las situaciones de interacción particulares explicaría, a su vez, la mayor variabilidad intrapersonal del docente en las diferentes clases a su cargo.

\section{Coincidencia de contenidos y de formulación lingüística}

Al margen del orden secuencial, es de interés analizar la correspondencia entre los contenidos de las clases y los del texto-fuente. Esto debe hacerse a un nivel temático más específico que el considerado hasta el momento, ya que una diferencia en este sentido sólo es perceptible en una dimensión más microscópica. En una escala temática y subtemática macroscópica no se registran diferencias muy visibles, esto es, casi todos los temas y subtemas del texto-fuente tienen referencia explícita en los distintos desarrollos didácticos. Por eso el análisis que sigue se remite a un subtema bien específico: el ítem B de la "Explicación física del vuelo de las aves". La Tabla 5 presenta los nueve subconceptos de dicho ítem $\mathrm{B}$ que explicita el texto-fuente y la información de si fueron aludidos o no en las diferentes clases. Para facilitar la relación entre coincidencia subconceptual y coincidencia lingüística que sigue, sólo se analiza la clase 1. 
TABLA 5. Subconceptos del ítem B del tema "Explicación física del vuelo de las aves" que fueron considerados u omitidos en el desarrollo de la clase 1 .

\begin{tabular}{|c|c|c|c|c|}
\hline Subconceptos & Nancy (E) & Ana $(E)$ & Carlos (P) & Inés $(\mathrm{P})$ \\
\hline No vuelo en el vacío & $\mathrm{X}$ & $\mathrm{X}$ & $\mathrm{X}$ & $\mathrm{X}$ \\
\hline Estados de la materia & $\mathrm{X}$ & $\mathrm{X}$ & $\mathrm{X}$ & $\mathrm{X}$ \\
\hline $\begin{array}{l}\text { Agua: líquido } \\
\text { Aire: gas (ambos son materia) }\end{array}$ & $\mathrm{X}$ & $\mathrm{X}$ & $\mathrm{X}$ & $\mathrm{X}$ \\
\hline Formados por moléculas & $\mathrm{X}$ & $\mathrm{X}$ & & \\
\hline Tienen peso y volumen & & $\mathrm{X}$ & & \\
\hline $\begin{array}{l}\text { Son fluidos } \\
\text { (estructura más flexible) }\end{array}$ & $\mathrm{X}$ & $\mathrm{X}$ & $\mathrm{X}$ & $\mathrm{X}$ \\
\hline $\begin{array}{l}\text { Ave: sólido en interacción con } \\
\text { materia gaseosa }\end{array}$ & $\mathrm{X}$ & $\mathrm{X}$ & $\mathrm{X}$ & $\mathrm{X}$ \\
\hline Total & 6 & 7 & 5 & 5 \\
\hline
\end{tabular}

Aunque las diferencias no son grandes, los docentes expositivos tienden a tener una mayor coincidencia subconceptual con el texto-fuente. La misma tendencia se aprecia cuando se analizan las coincidencias específicamente lingüísticas de los docentes con las estructuras expresivas más notorias (referidas al ítem B) que aparecen en el texto-fuente. Debe tenerse en cuenta que se trata de coincidencias de palabras y estructuras lingüísticas (las más salientes de cada subconcepto), y no sólo de contenido conceptual.

TABLA 6. Coincidencia lingüística entre el discurso del docente y el del texto-fuente referida al ítem B del tema "Explicación física del vuelo de las aves" en la clase 1.

\begin{tabular}{|c|c|c|c|c|}
\hline Estructuras lingüísticas & Nancy (E) & Ana $(E)$ & Carlos (P) & Inés $(\mathrm{P})$ \\
\hline "El ave no es un cuerpo material que vuela en el vacío" $\left({ }^{\circ}\right)$ & $\mathrm{X}$ & $\mathrm{X}$ & & $\mathrm{X}$ \\
\hline "La materia puede tener 3 estados" $\left({ }^{\circ}\right)$ & $\mathrm{X}$ & $\mathrm{X}$ & $\mathrm{X}$ & $\mathrm{X}$ \\
\hline "El aire es un gas" $\left({ }^{\circ}\right)$ & $\mathrm{X}$ & $\mathrm{X}$ & $\mathrm{X}$ & \\
\hline "Está formado por moléculas" & $\mathrm{X}$ & $\mathrm{X}$ & & \\
\hline "Tiene peso y volumen" & & $\mathrm{X}$ & & \\
\hline "Impresiona nuestros sentidos" & & $\mathrm{X}$ & & \\
\hline "Los líquidos y los gases son fluidos" $\left({ }^{\circ}\right)$ & $\mathrm{X}$ & $\mathrm{X}$ & & $\mathrm{X}$ \\
\hline "Tiene estructura más flexible, más libre e informe" $\left({ }^{\circ}\right)$ & & $\mathrm{X}$ & & \\
\hline "Interrelación entre un cuerpo sólido y un cuerpo gaseoso que lo rodea" $\left({ }^{\circ}\right)$ & $\mathrm{X}$ & & & \\
\hline TOTAL & 6 & 8 & 2 & 3 \\
\hline
\end{tabular}

$\left.{ }^{\circ}\right)$ : Estructuras lingüísticas correspondientes a subconceptos aludidos por todos los docentes. 
También aquí se reitera la tendencia de la tabla anterior, ya que los profesores expositivos manifiestan mayores coincidencias lingüísticas con el texto-fuente que sus colegas participativos. Esto es significativo por cuanto no se trata sólo de una mayor dependencia respecto a contenidos, sino también a nivel de la formulación lingüística de los mismos. Incluso si se restringe el análisis lingüístico a los ítems subconceptuales que fueron aludidos por todos los docentes $\left(^{\circ}\right)$, para evitar el solapamiento entre ambos tipos de análisis, la tendencia de una mayor dependencia lingüística respecto del texto-fuente es más notoria en el estilo expositivo: 5 y 5 en el casos de los docentes expositivos, y 2 y 3 en el caso de los profesores participativos.

\section{TEMAS EMERGENTES NO CONTEMPLADOS EN EL TEXTO-FUENTE}

Con todo, este análisis resultaría incompleto si no se aludiera a otro fenómeno colateral: los temas de emergencia espontánea que aparecen en el desarrollo de las clases, ya sea aportados por el docente o por los alumnos. Los temas más frecuentes que emergieron espontáneamente en las clases (ciertamente mucho más en las clases participativas) fueron los siguientes.

- Analogía ave-avión en la explicación física del vuelo.

Nancy (E) clase 1 y 2, Ana (E) clase 1, Carlos (P) clase 1 y 2, Inés (P) clase 1 y 2.

- Referencia a la explicación del vuelo de un globo aerostático.

Carlos (P) clase 1, Inés (P) clase 1 y 2.

- Referencia explícita a la ley de gravitación universal.

Carlos (P) clase 1 y 2, Inés (P) clase 2.

- Otras funciones de las plumas, cortejo sexual, ahuyentar a otras aves, defensa.

Carlos (P) clase 2, Inés (P) clase 1 y 2.

- Diferencias funcionales entre las alas y las extremidades superiores de los humanos. Carlos (P) clase 2, Inés (P) clase 1 y 2.

- Otros ejemplos de adaptación morfológica al medio en las aves y en otras especies. Nancy (E) clase 1, Ana (E) clase 2, Carlos (P) clase 1 y 2, Inés (P) clase 1 y 2.

- Otros ejemplos de adaptación dinámica al medio en las aves y en otras especies.

Ana (E) clase 2, Carlos (P) clase 1 y 2 , Inés $(\mathrm{P})$ clase 1 y 2.

Claramente, los temas emergentes fueron más frecuentes en las clases participativas, sea aportados espontáneamente por los alumnos y retomados por el docente, sea introducidos directamente por este. Sólo la referencia analógica al vuelo del avión y algunos ejemplos originales de adaptación morfológica al medio (el mimetismo de los insectos y el pico del picaflor) tuvieron referencia explícita, por parte del profesor, en las clases expositivas. En las clases participativas emergieron, además, los restantes temas. Este fenómeno tiene que ver sin duda con la mayor independencia y flexibilidad epistémica que parece ser un rasgo de este estilo de enseñanza. 


\section{DISCUSIÓN}

El corpus analizado estuvo constituido por ocho clases, a cargo de cuatro docentes. Si además se tiene en cuenta que el análisis sólo se focalizó en torno a dos subtemas, es claro que no hay margen para la inferencia estadística. Sólo es lícito hablar de tendencias constatables en los resultados. Sin embargo, estas tendencias son lo suficientemente evidentes como para pensar que trascienden la casuística y admiten un grado razonable de generalización. Al fin de cuentas, esto es a lo que se aspira en las investigaciones naturalistas de casos paradigmáticos.

Los resultados muestran con claridad que el estilo expositivo es intraindividualmente más constante, lo cual equivale a decir que el estilo participativo es intraindividualmente más variable. La explicación más plausible de este fenómeno remite a la mayor dependencia situacional del desarrollo didáctico que es propia de dicho estilo. En este caso, el desarrollo epistémico está condicionado por las vicisitudes de la interacción con los alumnos. El marco epistémico marca una orientación, pero el desarrollo de la enseñanza está prioritariamente determinado por el particular contexto interactivo.

El otro aspecto analizado, que representa la contracara del primero, es el de la dependencia del docente respecto de la fuente epistémica ofrecida. Aquí los resultados muestran con claridad que el estilo expositivo es el que más lo sigue de cerca, asumiendo el texto-fuente una función normativa central. Esto no ocurre con el estilo participativo, donde se aprecia una mayor independencia y flexibilidad respecto a este. Este fenómeno es perfectamente entendible a la luz de la escasa interacción (comunicación unilateral) que es propia del estilo expositivo, centrado esencialmente en la episteme que se debe enseñar.

Estando el discurso didáctico a cargo exclusivamente del experto, una ventaja intrínseca del estilo expositivo es la mejor organización y sistematización del conocimiento enseñado. En el estilo participativo hay una verdadera negociación o co-construcción de significado (Coll y Onrubia, 1996; Cubero, 2005), lo cual implica una dosis de impredecibilidad y de singularidad, y un desarrollo más fragmentado (por episodios más que unitario). La ventaja intrínseca de este tipo de desarrollo didáctico es el de generar un conocimiento más flexible y transferible, puesto de manifiesto en la aparición de temas emergentes o espontáneos. También hay que destacar la mejor integración con el saber pre-existente de los alumnos, lo que tiene mucha importancia en relación al cambio conceptual.

En la introducción ya se ha aludido a la crítica de Kirschner et al. (2006), a la instrucción que se aparta de la instrucción directamente guiada, en la que el experto cede el protagonismo frente a la actividad de los alumnos (lo cual no es del todo aplicable al presente caso por cuanto los docentes en ningún caso renunciaron a guiar el desarrollo epistémico). La base de dicha crítica es la distinción neta que hacen los autores entre memoria de trabajo (activada en el proceso de enseñanza) y memoria de largo plazo (activada en la recuperación del conocimiento aprendido). El estilo participativo requeriría una fuerte carga cognitiva para hacer frente al proceso de negociación epistémica, que es complejo porque implica atender dos dimensiones: la específicamente epistémica y la articulación social entre los múltiples agentes intervinientes (lo que algunos llaman dimensión de tarea y dimensión de mantenimiento). Con todo, esta inversión de trabajo cognitivo en la enseñanza no 
garantizaría en modo alguno el aprendizaje, es decir, la recuperación del conocimiento a largo plazo (más allá de la situación de enseñanza).

En consonancia con la tesis de Kirschner et al. referida, la crítica al constructivismo radical ha hecho notar que la introducción de información nueva (elemento central de la enseñanza) es básicamente una función experta, es decir, de quien se supone conoce el conocimiento que se quiere enseñar; la interacción y el intercambio con los alumnos sólo podría hacerse en torno a la información ya conocida (su función básica sería la inserción del conocimiento nuevo en el saber pre-existente). Es claro que el estilo expositivo pone el acento en la información nueva, mientras que el estilo participativo debe ocuparse de ambas.

Por supuesto, uno podría preguntarse, cómo lo hace Kuhn (2007), cuál es el objetivo de la enseñanza o qué es exactamente aprender un conocimiento ("aprendizaje para qué", "aprendizaje para quién”). Además del aspecto relativo al conocimiento, el aprendizaje supone un traspaso del experto al alumno del control de la actividad, como bien lo hacen notar Coll y Onrubia (1996). Esto tiene que ver no solamente con la apropiación activa y personal del conocimiento, sino con el desarrollo de mecanismos más generales de autorregulación ("aprender a aprender"), que van más allá del aprendizaje de temas específicos. Es razonable suponer que en esto el estilo participativo estaría en ventaja. De todos modos, en este trabajo no se consideran los datos del aprendizaje logrado con ambos estilos.

Otro aspecto que merece referencia es la cuestión motivacional, que no pudo medirse comparativamente por tratarse de "muestras" independientes. Diversos autores (Coldren y Hively, 2009; Hood et al., 2009; Pierro et al., 2009) sostienen que el estilo participativo genera más motivación en los alumnos. Es probable que el clima de clase, más distendido en el estilo participativo, guste más a los alumnos. Sin embargo hay que ser muy prudentes en esto, ya que los alumnos (sobre todo los de nivel secundario y universitario) priorizan en sus valoraciones la calidad de la enseñanza y personal del docente (incluido el buen trato y el carácter afable), por sobre la modalidad de enseñanza. El estilo expositivo no implica necesariamente autocratismo y frialdad afectiva. Los cuatro docentes que participaron del estudio eran considerados "buenos profesores", tanto por la calidad docente como por el trato con los alumnos.

Con todo hay que reconocer ciertas diferencias que forman parte del estilo de enseñanza asumido. Como lo señala Stacy (2009), el estilo expositivo es más retórico, con una sintaxis más cuidada. Se diría que se trata de una oralidad cercana a la escritura. El destinatario de su comunicación es un sujeto colectivo, al punto que algunos han constatado que la mirada es generalizada, sin focalizarse en sujetos individuales. También se ha señalado (Reeve, 2009) que su campo espacial es el frente de la clase, con pocos desplazamientos por el salón de clase.

Otro aspecto que se debe tener en cuenta, cuando se habla de estilos, es que estos no son independientes de las tradiciones institucionales ("cultura de la escuela") y de los hábitos y expectativas de los alumnos. No todos los ámbitos educativos recepcionan de la misma manera las modalidades de enseñanza, y el docente no deja de estar atento a estas expectativas, las más de las veces implícitas. Pero esto no impide hablar de estilos de enseñanza, que implican disposiciones de una cierta estabilidad respecto a la manera de encarar la actividad de enseñar. 
Es precisamente el uso del concepto estilo de enseñanza lo que algunos pueden criticar. Al fin y al cabo, podrán decir, el dispositivo empírico de la investigación consiste simplemente en comparar clases de distinto tipo: expositivas y participativas. No se comparan unidades didácticas, es decir, segmentos molares de desarrollo didáctico que implican varias clases y tipos de actividad, como lo proponen Coll y Onrubia (1994). La respuesta pasaría por recordar que los docentes participantes fueron seleccionados por su respectiva modalidad estable de enseñar. Razones de factibilidad determinaron restringir la comparación a unidades-clase, eligiéndose la exposición y la interacción-guiada como las actividades más paradigmáticas de cada estilo. Esto no implica desconocer que en los desarrollos de unidades didácticas molares suelen coexistir actividades expositivas y de participación-guiada. La escisión entre estos dos tipos de actividad fue un recurso metodológico, lícito en virtud de lo que los escenarios naturales muestran: que hay profesores más proclives a la exposición y otros más inclinados a la interacción con los alumnos. Lo que metodológicamente se ha hecho fue separar completamente ambas actividades de modo de evitar la hibridez de las condiciones objeto de comparación.

Otro aspecto pasible de crítica es unificar bajo el rótulo de participación-guiada modalidades de enseñanza muy diversas que suelen caer dentro de la megacategoría "constructivismo". Precisamente, se evitó esta etiqueta cuyo peso conceptual trasciende el término menos ideológico y más empírico de "estilo de enseñanza". De acuerdo a nuestra definición operacional, el estilo expositivo es el que se caracteriza por el uso privilegiado de la explicación magistral, en tanto que el estilo participativo-guiado es el que promueve el diálogo socioconstructivo con los alumnos garantizando la guía epistémica constante del docente.

\section{CONCLUSIONES}

1. Corresponde reconocer que futuras investigaciones deberían atender dos variables intervinientes importantes: el nivel de escolaridad y los distintos contenidos epistémicos.

2. También podrían abordarse unidades didácticas más amplias, aspectos comportamentales no exclusivamente discursivos, y actividades didácticas no limitadas a la mera exposición o al diálogo-guiado con el grupo clase. De hecho, en las clases implementadas hubo muchas otras actividades asociadas al discurso docente, como por ejemplo el uso del pizarrón, power-point o material pictórico. La pregunta que cabe es si estas actividades están asociadas a un determinado estilo de enseñanza o si simplemente son variables independientes que pueden estar generando un efecto parásito. La única manera de contestar esta pregunta es aumentando la cantidad de casos considerados.

3. Un estudio expost-facto dirigido a relevar observacionalmente en clases naturales datos sobre modalidades de enseñanza y actividades que componen el desarrollo didáctico sería sin duda de gran ayuda en este sentido. No fue el objetivo de la presente investigación, la que apuntó exclusivamente a analizar la constancia-variabilidad del discurso docente y la dependencia-independencia de este respecto de la fuente epistémica. 


\section{REFERENCIAS BIBLIOGRÁFICAS}

1. Astolfi, J.P. (1997). Tres modelos de enseñanza. En J. P. Astolfi: Aprender en la Escuela. Chile: Dolmen, 127-135.

2. Aubert, A., García, C. \& Racionero, S. (2009). El aprendizaje dialógico. Cultura y Educación, vol. 21 (2), 128-140.

3. Ausubel, D. P., Novak, J. D. \& Hanesian, H. (1983). Psicología educativa. México: Trillas.

4. Beltrán, J. (1998). Concepto, desarrollo y tendencias actuales de la psicología de la instrucción. En J. Beltrán y C. Genovard (eds.): Psicología de la Instrucción I. Madrid: Síntesis. Cap. 1, 19-86.

5. Bennett, N. (1998). Investigaciones recientes sobre los procesos de enseñanza y aprendizaje en el aula. En M. Carretero (comp.): Procesos de Enseñanza y Aprendizaje. Buenos Aires: Aique.

6. Bennett, N. (1979). Estilos de Enseñanza y Progreso de los Alumnos. Madrid: Morata.

7. Cardozo, S., Andino, G., Brunnetti Esquivel, A. \& Espindola, E. (2008). Efectividad de los métodos activos como estrategia de enseñanza-aprendizaje en grupos grandes y heterogéneos. Revista Cubana de Educación Superior [on line], vol. 22 (1), pp. 0-0. ISSN 0864-2141.

8. Carretero, M. (1998). La investigación europea sobre enseñanza y aprendizaje. En M. Carretero (comp.): Procesos de Enseñanza y Aprendizaje. Buenos Aires: Aique.

9. Coldren, J. \& Hively, J. (2009). Interpersonal teaching style and student impression formation. College Teaching, vol. 57 (2), 93-98.

10. Coll, C. \& Onrubia, J. (1996). La construcción de significados compartidos en el aula: actividad conjunta y dispositivos semióticos en el control y seguimiento mutuo entre profesor y alumno. En C. Coll y D. Edwards, Enseñanza, Aprendizaje y Discurso en el Aula. Madrid: Fund. Infancia y Aprendizaje, 53-74.

11. Coll, C. \& Onrubia, J. (1994). Temporal dimension and interactive processes int teaching-learning activities: a theoretical and methodological challenge. En N. Mercer y C. Coll (eds.), Teaching, Learning and Interaction. Madrid: Fund. Infancia y Aprendizaje, 107-122.

12. Coll, C., Onrubia, J. \& Maurí. T. (2008). Ayudar a aprender en contextos educativos: el ejercicio de la influencia educativa y el análisis de la enseñanza. Revista de Educación, N. ${ }^{\circ} 346,33-70$.

13. Coll, C. \& Sánchez, E. (2008). Presentación. El análisis de la interacción alumno-profesor: líneas de investigación. Revista de Educación, N. ${ }^{\circ} 346,15-32$.

14. Cubero, R. (2005). Perspectivas Constructivistas. Barcelona: Graó. 
15. Díaz Arce, T. (2003). La interpretación histórico-cultural de la transposición didáctica como puente de emancipación del aprendizaje y la enseñanza. Revista Praxis (Chile), N. ${ }^{\circ} 3,37-56$.

16. Flanders, N. (1960). Teacher Influences, Pupil Attitudes and Achievement. Ann Arbor: University of Michigan.

17. García Pérez, F. (2000). Docencia, 3 (12), 21-34. Santiago de Chile: Colegio de Profesores de Chile.

18. Gargallo López, B. (2008). Estilos de docencia y evaluación de los profesores universitarios y su influencia sobre los modos de aprender de sus estudiantes. Revista Española de Pedagogía, 66 (241), 425-446.

19. Gargallo López, B., Suárez Rodríguez, J. \& Ferreras Remesal, A. (2007). Estrategias de aprendizaje y rendimiento académico en estudiantes universitarios. Revista de Investigación Educativa, 25 (2), 421-441.

20. González Peiteado, M. (2009). Estilos de enseñanza predominantes en los alumnos de los centros de formación del profesorado de la provincia de Pontevedra. Innovación Educativa, 19, 237-245.

21. Hood, J., Poulson, R., Mason, S., Walker, T. \& Dixon, J. (2009). An examination of traditional and nontraditional students' evaluations of professorial leardership styles: transformational versus transactional approach. Journal of the Scholarship of Teaching and Learning, vol. 9 (1), 1-12.

22. Jones, V., Holland, A. \& Oldmeadow, W. (2008). Inductive teaching method - An alternate method for small group learning. Medical Teacher, 30 (8), 246-249.

23. Kirschner; P., Sweller, J. \& Clark, R. (2006). Why minimal guidance during instruction does not work: an analysis of the failure of constructivist, discovery, problem-based, experiential, and inquiry-based teaching. Educational Psychologist, 41 (2), 75-86.

24. Kozulin, A. (2004). Vygotsky's theory in the classroom: introduction. European Journal of Psychology of Educatión, vol. 19 (1), 3 - 7.

25. Kuhn, D. (2007). Is direct instruction an answer to the right question?. Educational Psychologist, vol. 42 (2), 109-113.

26. Leyens, J. Ph. (1989). Psychologie Sociale. Bruxelles: Mardaga.

27. Marín Gracia, Ma. A. (2002). La investigación sobre diagnóstico de los estilos de aprendizaje en la enseñanza superior. Revista de Investigación Educativa, 20 (2), 303-337.

28. Michel, N., James, J. \& Varela, O. (2009). Active versus passive teaching styles: an empirical study of student learning outcomes. Human Resource Development Quarterly, vol. 20 (4), 397-418.

29. Monereo, C. (2006). Estrategias de Enseñanza y Aprendizaje. Barcelona: Graó. 
30. Mugny, G., Chatard, A. \& Quiamzade, A. (2006). The social transmission of knowledge at the university: teaching style and epistemic dependence. European Journal of Psychology of Education, vol. 21 (4), 413-427.

31. Onrubia, J. (1996). Ayuda y ajuste de la ayuda: la enseñanza como proceso de creación de zonas de desarrollo próximo y de asistencia en ellas. En C. Coll (ed.): El Constructivismo en el Aula, Barcelona: Graó. Cap. 5, 101-124.

32. Pierro, A., Presaghi, F., Higgins, T. \& Kruglanski, A. (2009). Regulatory mode preferentes for autonomy supporting versus controlling instructional styles. British Journal of Educational Psychology, 79, 599-615.

33. Pinelo Ávila, F. (2008). Estilos de enseñanza de los profesores de la carrera de psicología. Revista REMO (México), vol. 5 (13), 17-24.

34. Pozo, J. I. \& Gómez Crespo, M. A. (1998). Aprender y enseñar ciencia. Madrid: Morata.

35. Quaas, C. \& Crespo, N. (2003). ¿Inciden los métodos de enseñanza del profesor en el desarrollo del conocimiento metacomprensivo de sus alumnos? Revista Signos, vol. $36(54), 225-234$.

36. Quiamzade, A. \& Mugny, G. (2009). When teaching style matches students' epistemic (in)dependence: the moderating effect of perceived epistemic gap. European Journal of Psychology of Education, vol. 24 (3), 361-371.

37. Reeve, J. (2009). Why teachers adopt a controlling motivating style toward students and how they can become more autonomy supportive. Educational Psychologist, 44 (3), 159-175.

38. Roselli, N. (2010). Comparación experimental entre tres modalidades de enseñanza mediadas informáticamente. Revista de Investigación Educativa, 28 (2), 265-282

39. Roselli, N. (2007). La eficacia comparativa de tres modalidades instruccionales paradigmáticas. Revista de Psicología (UCA, Buenos Aires), Vol. 3 (2).

40. Roselli, N. (2003) Modalidades de tutoría docente en la resolución de problemas en el aprendizaje de conocimientos. Versión ampliada del artículo homónimo. Cuadernos de la Facultad de Cs. de la Educación de la Universidad Nacional de Entre Ríos, Paraná.

41. Ruiz Lara, E., Hernández Pina, F. \& Ureña Villanueva, F. (2008). Enfoques de aprendizaje y rendimiento institucional y afectivo de los alumnos de la titulación de cs. de la actividad física y del deporte. Revista de Investigación Educativa, 26 (2), 307-322.

42. Schwerdt, G. \& Wuppermann, A. (2009). Is traditional teaching really all that bad? A within-student between-subject approach. Cesifo Working Paper 2634. Munich: CESifo.

43. Stacy, J. (2009). The guide on the stage: In defense of good lecturing in the history classroom. Social Education, vol. 73 (6), 275-278. 
44. Toczek, M. \& Morge, L. (2009). Effects of evaluative vs. co-constructive interactions on learnig in physics. European Journal of Psychology of Education, vol. 24 (3), 325-333.

45. Weber, E. (1976). Estilos de Educación. Barcelona: Herder.

46. Weltman, D. \& Whiteside, M. (2010). Comparing the effectiveness of tradicional and active learning methods in business stadistics: Convergence to the mean. Journal of Stadistics Education, vol. 18 (1).

47. Yildirim, O., Acar, A., Bull, S. \& Sevinc, L. (2008). Relationships between teacher's percieved leaderships style, student's learning style, and academic achievement: A study on high school students. Educational Psychology, vol. (28) (1), 73-81. 